\title{
On the ground state energy of the linear antiferromagnetic Heisenberg chain with nearest- and next-nearest-neighbour interactions
}

\author{
P M van den Broek \\ Department of Applied Physics, Twente University of Technology, Enschede, The \\ Netherlands
}

Received 18 June 1980

\begin{abstract}
We calculate lower bounds for the ground state energy of the linear antiferromagnetic Heisenberg chain with nearest- and next-nearest-neighbour interactions. From these lower bounds new upper bounds are derived. Several approximations of the ground state energy given in the literature are compared with these upper and lower bounds. It is found that the best approximation is given by extrapolation from finite cyclic chains. This extrapolation, however. is restricted to certain values of the relative strength of the interactions. Here we extrapolate the ground state energy from finite open chains where no such restriction occurs.
\end{abstract}

\section{Introduction}

We consider the linear antiferromagnetic Heisenberg chain with nearest- and nextnearest-neighbour interactions with Hamiltonian

$$
H_{N}(\gamma)=\sum_{i=1}^{N-1} \sigma_{i} \cdot \sigma_{i+1}+\gamma \sum_{i=2}^{N-2} \sigma_{i} \cdot \sigma_{i+2}
$$

where $N$ is the number of spins $\frac{1}{2}$ in the chain. Let the lowest eigenvalue of $H_{N}(\gamma)$ be $E_{N}(\gamma)$. We are interested in the energy per spin in the ground state in the limit of large $N$

$$
E(\gamma)=\lim _{N \rightarrow \infty} E_{N}(\gamma) / N
$$

For $\gamma=0$ and $\gamma=\frac{1}{2}$ the ground state energy is known exactly: $E(0)=-1-4 \ln 2=$ -1.7726 (Hulthén 1938); $E\left(\frac{1}{2}\right)=-1.5$ (van den Broeck 1980). Approximations for general $\gamma$ have been given by Ono (1972), Ananthakrishna et al (1976), van de Braak et al (1977) and van den Broek et al (1980). Upper bounds have been given by Majumdar and Ghosh (1969a), Niemeyer (1971), van de Braak et al $(1977,1979)$ and van den Broek et al (1980).

In $\S 2$ we derive lower bounds for $E(\gamma)$. From these lower bounds new upper bounds are derived in $\S 3$. In $\S 4$ the various approximations are compared with the lower and upper bounds. It turns out that the best approximation is given by extrapolation from finite cyclic chains (Ono 1972 and Ananthakrishna et al 1976). This extrapolation, however, can not be made for $\gamma>\frac{1}{2}$. In $\S 5$ an extrapolation is given from finite open chains 
for $\gamma$ in the interval $[-1,1]$. The results are tabulated together with the best upper and lower bounds.

\section{Lower bounds}

Let $M$ be some integer, $M>2$ and define

$$
\begin{aligned}
H^{M, n}(\gamma)=\frac{1}{2} \sigma_{n} & \cdot \sigma_{n+1}+\frac{1}{2} \sigma_{n+M-2} \cdot \sigma_{n+M-1}+\sum_{i=1}^{M-3} \sigma_{n+i} \cdot \sigma_{n+i+1} \\
& +\gamma \sum_{i=1}^{M-2} \sigma_{n+i-1} \cdot \sigma_{n+i+1} .
\end{aligned}
$$

We suppose that $N-2$ is divisible by $M-2$; then

$$
H_{N}(\gamma)=\sum_{n} H^{M, n}(\gamma)+\frac{1}{2} \sigma_{1} \cdot \sigma_{2}+\frac{1}{2} \sigma_{N-1} \cdot \sigma_{N}
$$

where the summation is over $n=1, M-1,2 M-3, \ldots, N-M+1$. So we have split up $H_{N}(\gamma)$ into $(N-2) /(M-2)$ identical parts corresponding to $(N-2) /(M-2)$ overlapping cells of $M$ spins, and two boundary terms. This enables us to compute lower bounds for $E_{N}(\gamma)$ since the lowest eigenvalue of a sum of operators is not less than the sum of the lowest eigenvalues of these operators. We thus have

$$
E_{N}(\gamma) \geqslant \frac{N-2}{M-2} E^{(M)}(\gamma)-3
$$

where $E^{(M)}(\gamma)$ is the lowest eigenvalue of $H^{M, n}(\gamma)$ (which is of course independent of $n$ ). Dividing by $N$ and taking the limit $N \rightarrow \infty$ gives

$$
E(\gamma) \geqslant \frac{E^{(M)}(\gamma)}{M-2}
$$

$E^{M}(\gamma)$ can be calculated by diagonalising $H^{M, n}(\gamma)$; the largest $M$ we were able to handle was $M=8$. It turned out that for most values of $\gamma$ the best lower bound is obtained with $M=7$. Let us denote the lower bound obtained in this way by $E_{\mathrm{LB}}(\gamma)$

$$
E_{\mathrm{LB}}(\gamma)=\operatorname{Min}_{3 \leqslant M \leqslant 8} \frac{E^{(M)}(\gamma)}{M-2}
$$

This lower bound can be improved using the fact that $E(\gamma)$ is a concave function of $\gamma$. That $E(\gamma)$ is a concave function can be seen as follows. Let $|\psi\rangle$ be the ground state of $H_{N}\left(\gamma_{0}\right)$ for some $\gamma_{0}$

$$
H_{N}\left(\gamma_{0}\right)|\psi\rangle=E_{N}\left(\gamma_{0}\right)|\psi\rangle
$$

Then

$$
E_{N}(\gamma) \leqslant\left\langle\psi\left|H_{N}(\gamma)\right| \psi\right\rangle=E_{N}\left(\gamma_{0}\right)+\left(\gamma-\gamma_{0}\right)\left\langle\psi\left|\sum_{i=1}^{N-2} \sigma_{i}, \sigma_{i+2}\right| \psi\right\rangle .
$$

The right-hand side of this equation is an upper bound for $E_{N}(\gamma)$ and a tangent line with tangent point $\gamma_{0}$. So $E_{N}(\gamma)$, and thus $E(\gamma)$, is a concave function. In the same way $E^{(M)}(\gamma)$ is also a concave function of $\gamma$.

From the fact that $E(\gamma)$ is a concave function it follows that the tangent lines of $E_{\mathbf{L B}}(\gamma)$ 
Table 1.

\begin{tabular}{cllll}
\hline$\gamma$ & Lower bound & \multicolumn{2}{c}{ Upper bound } & Extrapolation \\
\hline-1.0 & -2.7035 & -2.4891 & $(4)$ & -2.5944 \\
-0.9 & -2.6105 & -2.4080 & $(4)$ & -2.5068 \\
-0.8 & -2.5174 & -2.3270 & $(4)$ & -2.4199 \\
-0.7 & -2.4243 & -2.2459 & $(4)$ & -2.3338 \\
-0.6 & -2.3312 & -2.1649 & $(4)$ & -2.2488 \\
-0.5 & -2.2381 & -2.0838 & $(4)$ & -2.1648 \\
-0.4 & -2.1450 & -2.0027 & $(4)$ & -2.0824 \\
-0.3 & -2.0519 & -1.9413 & $(3)$ & -2.0015 \\
-0.2 & -1.9588 & -1.8851 & $(3)$ & -1.9225 \\
-0.1 & -1.8657 & -1.8288 & $(3)$ & -1.8457 \\
0 & -1.7726 & -1.7726 & $(1)$ & -1.7715 \\
0.1 & -1.7163 & -1.6795 & $(3)$ & -1.7004 \\
0.2 & -1.6601 & -1.5864 & $(3)$ & -1.6331 \\
0.3 & -1.6038 & -1.5437 & $(5)$ & -1.5714 \\
0.4 & -1.5476 & -1.5126 & $(5)$ & -1.5216 \\
0.5 & -1.5 & -1.5 & $(2)$ & -1.5 \\
0.6 & -1.5544 & -1.5154 & $(5)$ & -1.5246 \\
0.7 & -1.6617 & -1.5624 & $(5)$ & -1.5852 \\
0.8 & -1.7848 & -1.6361 & $(5)$ & -1.6695 \\
0.9 & -1.9620 & -1.7304 & $(5)$ & -1.8011 \\
1.0 & -2.1393 & -1.8408 & $(5)$ & -2.0064 \\
\hline
\end{tabular}

(1) Exact value; Hulthén (1938).

(2) Exact value; van den Broek (1980).

(3) This paper.

(4) Niemeyer (1971).

(5) van den Broek et al (1980).

which go through the exact value $E(0)=-1.7726$ are lower bounds for $E(\gamma)$ in the region between the tangent point and 0 . These lower bounds improve the lower bound $E_{L B}(\gamma)$. From the other exact value $E\left(\frac{1}{2}\right)=-1.5$ no new lower bounds can be obtained since $E_{\mathrm{LB}}\left(\frac{1}{2}\right)=-1 \cdot 5$.

The slope of the function $E(\gamma)$ is decreasing monotonically from +1 until -1.7726 . This means that the tangent line to the curve $E_{\mathrm{LB}}(\gamma)$ with slope -1.7726 gives an improved lower bound in the region right from the tangent point. A tangent line with slope +1 cannot be drawn since the slope of $E_{\mathrm{LB}}(\gamma)$ goes to +1 from below if $\gamma \rightarrow-\infty$.

The lower bound obtained in this way is given in table 1 for several values of $\gamma$ between -1 and +1 . This lower bound is better than the trivial lower bound given by Majumdar and Ghosh (1969a), which was the only lower bound in the literature until now.

\section{Upper bounds}

In the previous section we found that the tangent lines of $E_{\mathrm{LB}}(\gamma)$ through the exact value $E(0)=-1.7726$ are lower bounds between 0 and the tangent points. The tangent line with negative tangent point is an upper bound for $y \geqslant 0$ and the tangent line with positive tangent point is an upper bound for $\gamma \leqslant 0$. This also follows from the fact that 
$E(\gamma)$ is a concave function. These upper bounds are better than the upper bounds in the literature for values of $\gamma$ in the neighbourhood of 0 .

In table 1 we present for some values of $\gamma$ between -1 and 1 the best upper bound for $E(\gamma)$ known until now. The numbers between brackets indicate where the upper bound has been derived:

\section{Discussion}

The several approximations for $E(\gamma)$ existing in the literature may now be compared with the upper and lower bounds of table 1 . Here we will give for each approximation the number of values of $\gamma$ for which the approximated value of $E(\gamma)$ lies between the lower and upper bound. The number of values of $\gamma$ taken into account is 21.

Table 2.

\begin{tabular}{llc}
\hline Reference & Method & No of $\gamma$ \\
\hline $\begin{array}{l}\text { van de Braak et al }(1977) \\
\text { van den Broek et al }(1980)\end{array}$ & Renormalisation method & 1 \\
& Perturbation theory; second order: 2 spins per cell & 3 \\
third order: 2 spins per cell & 4 \\
Majumdar and Ghosh $(1969 \mathrm{~b})$ & second order: 4 spins per cell & 11 \\
Ananthakrishna et al $(1976)$ & Closed chain of 10 spins & 16 \\
This paper, $\$ 5$ & Extrapolation from finite closed chains & 13 \\
\hline
\end{tabular}

Here one should note that Ananthakrishna et al (1976) take only 15 of the 21 values of $\gamma$ into account $(-0.9 \leqslant \gamma \leqslant 0.5)$.

For these values of $\gamma$ their extrapolation method agrees very well with the upper and lower bounds: only the two exactly known results are not obtained. Because of this restriction in the range of values of $\gamma$ where the extrapolation method for cyclic chains works, we give in the next section an extrapolation from open chains, where we find no restriction on the range of values of $\gamma$; it turns out that for the 21 values of $\gamma$ considered all extrapolated values lie between the upper and lower bounds except for $\gamma=0$, where the exact result is not reproduced.

\section{Extrapolation from finite open chains}

The ground state energy per spin of finite open chains, $E_{N}(\gamma) / N$, is known for $N$ up to 8 . It turns out that $E(\gamma)-E_{N}(\gamma) / N$ is approximately proportional to $1 / N$, with the proportionality being different for odd and even $N$. This is in distinction with the case of finite closed cells, where proportionality with $1 / N^{2}$ is found. We restrict ourselves here to even $N$ because for even $N$ the results are better than the results for odd $N$; this is not surprising since we have four values for even $N(N=2,4,6,8)$ and only three values for odd $N(N=3,5,7)$. The values of $E_{N}(\gamma) / N$ for $N=2,4,6,8$ are given in van den Broek 
et al (1980) for all 21 values of $\gamma$ under consideration here. We expand $E_{N}(\gamma) / N$ in powers of $1 / N$

$$
E_{N}(\gamma) / N=\sum_{n=0}^{\infty} a_{n}(\gamma) / N^{n}
$$

and we assume that, in a good approximation, the series can be truncated after a few, in our case four, terms

$$
E_{N}(\gamma) / N=\sum_{n=0}^{3} a_{n}(\gamma) / N^{n} .
$$

For each $\gamma$ we have four linear equations (from $N=2,4,6,8$ ) from which we can solve $a_{0}, a_{1}, a_{2}$ and $a_{3}$. Then the extrapolated value of $E(\gamma)$ is $a_{0}(\gamma)$. The results of this procedure are given in the last column of table 1. All extrapolated values lie between the upper and lower bounds, except that (of course) for $\gamma=0$ the exact result of Hulthen has not been reproduced. In contradistinction to Ananthakrishna et al (1976) we do reproduce the exact result $E\left(\frac{1}{2}\right)=-1.5$, since $E_{N}\left(\frac{1}{2}\right) / N$ is equal to -1.5 for all $N$. The results for -0.9 $\leqslant \gamma \leqslant 0.5$ agree very well with the results of Ananthakrishna et al (1976); the differences are $0.6 \%$ and less.

\section{Acknowledgment}

It is a pleasure to thank Professor Dr W J Caspers for useful discussions.

\section{References}

Ananthakrishna G, Weiss L F, Foyt D C and Klein D C 1976 Physica B 81 275-84

Hulthén L 1938 Arkiv Math. Astron. Fys. 26A No. 11

Majumdar C K and Ghosh D K 1969a J. Math. Phys. 10 1388-98

—_ 1969b J. Math. Phys. 10 1399-1402

Niemeyer Th 1971 J. Math. Phys. 12 1487-91

Ono I 1972 Phys Lett. A 38 327-8

Van de Braak H P, Caspers W J, de Lange C and Willemse M W M 1977 Physica A 87 354-68

Van de Braak H P, Caspers W J, Gragert P K H and Willemse M W M 1979 J. Stat. Phys. $20487-97$

Van den Broek P M 1980 Phys. Lett. A 77 261-2

Van den Broek P M, Caspers W J and Willemse M W M 1980 Physica to be published 